\title{
Performance and future developments of the RHEA single-mode spectrograph
}

\author{
Joao Bento ${ }^{\mathrm{a}}$, Tobias Feger ${ }^{\mathrm{b}}$, Michael J. Ireland ${ }^{\mathrm{a}}$, Adam Rains ${ }^{\mathrm{a}}$, Nemanja Jovanovic ${ }^{\mathrm{c}}$, David W. \\ Coutts $^{\mathrm{b}}$, Christian Schwab ${ }^{\mathrm{b}}$, Alexander Arriola ${ }^{\mathrm{b}}$, and Simon Gross ${ }^{\mathrm{b}}$ \\ ${ }^{a}$ Research School of Astronomy and Astrophysics, Australian National University, Cotter \\ Road, Canberra, ACT, 2611, Australia \\ ${ }^{b}$ Department of Physics and Astronomy, Macquarie University, Sydney, NSW, 2109, Australia \\ ${ }^{\mathrm{c}}$ National Astronomical Observatory of Japan, Subaru Telescope, 650 North A'ohoku Place, \\ Hilo, HI, 96720, USA
}

\begin{abstract}
The Replicable High-resolution Exoplanet and Asteroseismology (RHEA) spectrograph is being developed to serve as a basis for multiple copies across a network of small robotic telescopes. The spectrograph operates at the diffraction-limit by using a single-mode fiber input, resulting in a compact and modal-noise-free unit. The optical design is mainly based on off-the-shelf available components and comprises a near-Littrow configuration with prism cross-disperser. The échelle format covers a wavelength range of 430-650 $\mathrm{nm}$ at $\mathrm{R}=75,000$ resolving power. In this paper we briefly summarize the current status of the instrument and present preliminary results from the first on-sky demonstration of the prototype using a fully automated 16 " telescope, where we observe stable and semi-variable stars up to $\mathrm{V}=3.5$ magnitude. Future steps to enhance the efficiency and passive stability of RHEA are discussed in detail. For example, we show the concept of using a multi-fiber injection unit, akin to a photonic lantern, which not only enables increased throughput but also offers simultaneous wavelength calibration.
\end{abstract}

Keywords: High-resolution, single-mode spectrograph, integral field unit, radial velocity, exoplanets

\section{INTRODUCTION}

Radial Velocity (RV) measurements for exoplanet detection can be limited by photon noise but more often by modal noise in multi-mode fibers in bright targets, which is typically attenuated but not eliminated by less than ideal fiber scrambling. This problem is particularly prominent in few mode fibers rather than high number of excited modes which are likely to be used in small telescopes. The use of multi-mode fibers in such applications is motivated by a high throughput but is fundamentally limited by the effects of poor guiding, seeing or PSF modeling issues. ${ }^{1}$ This problem extends even to highly coherent calibration sources through single to multi-mode fiber coupling, which in turn are typically used as PSF format estimators. ${ }^{2}$

One solution to this problem is to use uniquely single-mode fibers to feed stabilized spectrographs. These do not suffer from typical modal noise* and have a very well defined and constant PSF at the spectrograph injection, allowing for diffraction limited optical designs. As an additional advantage, the smaller pupil size leads to significantly smaller sized instruments, able to use off-the-shelf optical elements without risking offaxis aberrations. This, in turn, leads to much simpler temperature and pressure stabilization requirements as the volume of air that requires stabilization is much smaller. The major drawback is the loss of throughput when compared with multi-mode inputs, reducing the typical number of targets that can be observed by a large fraction. However, in principle, for non-photon limited observations, the ultimate RV precision achievable with single-mode fiber inputs surpasses that of multi-mode equivalents.

Further author information: (Send correspondence to J.Bento)

J.Bento.: E-mail: joao.bento@anu.edu.au, Telephone: +61 (0)2 61250227

${ }^{*}$ We note that polarization issues have been suggested to be potentially present and capable of affecting RV measurements $^{3}$

Ground-based and Airborne Instrumentation for Astronomy VI, edited by Christopher J. Evans, Luc Simard, Hideki Takami Proc. of SPIE Vol. 9908, 99086K · C 2016 SPIE · CCC code: 0277-786X/16/\$18 · doi: 10.1117/12.2232371 
With this in mind, we present the latest status, performance and planned future developments of the RHEA spectrograph. This instrument is a flexible single-mode fibre-fed échelle spectrograph designed to be stabilised for RV precision and to be replicable and inexpensive. In this paper we describe the optical design and the latest results from both laboratory and on-sky tests. Although its primary purpose is to be a prototype for many future spectrographs to be deployed on small $(<0.5 \mathrm{~m}$ aperture) robotic telescopes for long baseline RV monitoring of bright $(<V \sim 5$ magnitude) stars in search of Exoplanets and Asteroseismic signals, a copy has recently been deployed at the Subaru $8.2 \mathrm{~m}$ telescopes at Mauna Kea. This version is fed by a novel custom-made $3 \times 3$ formated array of single-mode fibres behind the SCExAO system, aiming to spectroscopically resolve stellar surfaces and outflows. ${ }^{4}$

In this paper we show that this instrument is capable of delivering few $\mathrm{m} / \mathrm{s}$ precision RV stability as well as forming the basis for a generation of planet finding spectrographs. In Section 2 we describe the design of the instrument. Section 3 describes a double enclosure temperature stabilization apparatus while Section 4 presents results associated with a test of the grating efficiency. Additionally, Section 5 shows preliminary data from on-sky observations and we discuss ongoing improvements and future work in Section 6. Finally, we summarize this paper in Section 7.

\section{INSTRUMENT DESIGN}

In this section we briefly describe the optical and mechanical designs for the current prototype of this instrument as well as some considerations regarding novel methods for ensuring maximum temperature and pressure stability on the required timescales.

The RHEA spectrograph is a cross-dispersed échelle spectrograph comprising of a double-pass near-Littrow configuration similar to that of the MIKE ${ }^{5}$ and $\mathrm{PFS}^{6}$ instruments. This minimizes the number of optical elements for the bulk of the instrument as well as reducing its size. The spectrograph is composed of off-the-shelf optical elements with the exception of the prism cross-disperser (made from high-dispersive N-SF5 glass), that provide a wavelength coverage of 430-650 nm, spread over 45 orders, at a resolution of $\lambda / \Delta \lambda \sim 75,000$. A minimum resolution of 50,000 was deemed to be required so as to resolve critical spectral features in K-type giant stars. The custom prism is designed to provide a suitable order separation required for a photonic lantern integration. A summary of the instrument characteristics of RHEA is given in Table 2. A more in-depth discussion of the optical design of the spectrograph can be found in a previous SPIE conference proceedings publication on RHEA. ${ }^{7}$

\begin{tabular}{|l|l|}
\hline Instrumental parameter & Measured value and vendor specification \\
\hline Spectrograph working aperture & $\mathrm{f} / 15.4$ \\
\hline Fiber-link & Nufern $460-\mathrm{HP}, 10 \mathrm{~m}, 0.13 \mathrm{NA}$ \\
\hline Beam size & $13 \mathrm{~mm}\left(1 / \mathrm{e}^{2}\right)$ \\
\hline Wavelength range & $430-650 \mathrm{~nm}$ \\
\hline Resolving power & $\lambda / \Delta \lambda \sim 75,000(550 \mathrm{~nm})$ \\
\hline Number of orders & 45 orders \\
\hline Pixel sampling & $2.0-2.9$ pixel $(430-600 \mathrm{~nm})$ \\
\hline Fiber relay system & $\begin{array}{l}\text { parabolic off-axis collimator, } \mathrm{f}=15 \mathrm{~mm} \\
\text { achromatic doublet, } \mathrm{f}=60 \mathrm{~mm}\end{array}$ \\
\hline Collimator / Camera & infinity-corrected tube lens, $\mathrm{f}=200 \mathrm{~mm}(\mathrm{f} / 6.7)$ \\
\hline Cross-disperser & $\mathrm{N}-$ SF5 prism, $40^{\circ}$ apex angle \\
\hline Vacuum window & $\mathrm{N}-$ BK7, $3^{\circ} 53^{\prime}$ apex angle \\
\hline Échelle grating & $\mathrm{R} 2,31.6 \mathrm{~g}$ mm ${ }^{-1}, 22.5 \times 45 \mathrm{~mm}$ ruled area \\
\hline CCD detector & Starlight Xpress Trius SX-694 2750x2200 $4.54 \mu \mathrm{m}$ pixels. \\
\hline
\end{tabular}

We note, however, that design choices have led to several minor component changes and that Table 2 shows the most up-to-date version of this instrument and the configuration used for the performance tests. The optical design of the instrument is displayed in Figure 1. 


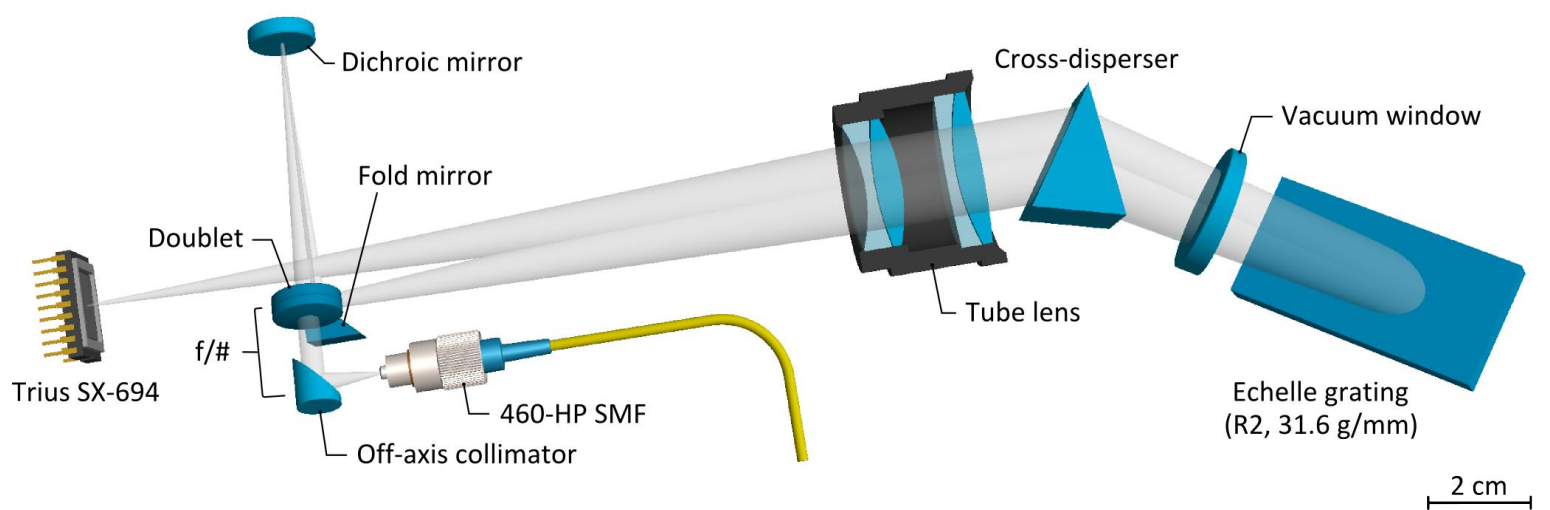

Figure 1. Optical layout of the RHEA spectrograph.

\section{THERMAL STABILITY}

The move from a lab environment to a typical small robotic telescope application implies a departure from an air conditioned and partly controlled room. The development of RHEA assumes copies of this spectrograph will be deployed on small robotic telescopes, likely inside the telescope dome itself, subjected to day/night temperature variability. As such, a suitable enclosure for the spectrograph itself is required. In this section we describe the several steps undertaken to achieve enough stability to guarantee that any level of variation can be compensated using a simultaneous wavelength calibration source.

A double enclosure system was constructed with the aim to maximize the isolation between the spectrograph mechanical structure and the dome environment. The primary enclosure consists of a foam insulated icebox (Evakool 47L) that provides a first order isolation. This box is then placed inside a second enclosure constructed from medium-density fiberboard (MDF) with an internal layer of Styrofoam. Figure 2 shows a schematic view of the insulating layers of the instrument (Left), as well as a photo of the instrument itself inside the primary enclosure. The final footprint of this instrument comes to $88 \times 66 \times 62 \mathrm{~cm}$.

The temperature stabilization system at the optical bench is done using a simple wire heater and controlled by a proportional-integral-derivative (PID) loop, actively closed using inputs from precision thermistors (RS 191-2128) mounted directly to various points of the aluminum frame. A second heating element for the outer enclosure is employed using a fan-heater (see Figure 2) also used to circulate air more efficiently. A proportionalintegral (PI) loop is then used to control the amount of heating required for this second insulation layer. Lab tests show that the temperature of the outer enclosure can be kept constant in a $\pm 0.1 \mathrm{~K}$ range and under moderately controlled conditions the optical bench has an rms variability on the temperature of under $5 \mathrm{mK}$. This is, however, not necessarily the case for dome environments where the typical day/night temperature variability can be as high as $15 \mathrm{~K}$, but any instrumental drifts due to this effect can be corrected for using simultaneous calibration.

\section{4. ÉCHELLE GRATING EFFICIENCY TESTS}

Throughput tests of the spectrograph have shown that the échelle grating exhibits an efficiency about half the assumed value. In order to quantify the poor performance, we assembled a test rig that allowed us to measure the absolute efficiency of the grating (in quasi-Littrow mode) by comparing the signal strength of the diffracted beam with the signal measured at the laser output (radiometric approach). The measurement is straightforward when using a single monochromatic line. For a constant angle of incidence $\alpha=63.4^{\circ}$, échelle order and wavelength, the diffraction angle $\beta$ becomes simply a function of the off-plane angle

$$
\beta(\gamma)=\sin ^{-1}\left(\frac{m \cdot \lambda}{\sigma_{e} \cos \gamma}-\sin \alpha\right)
$$

where $\gamma$ denotes the rotation (i.e. off-plane) angle around the Y-axis. A change in the off-plane angle causes a change in the diffraction angle which in turn translates the emission line through the FSR of a particular 


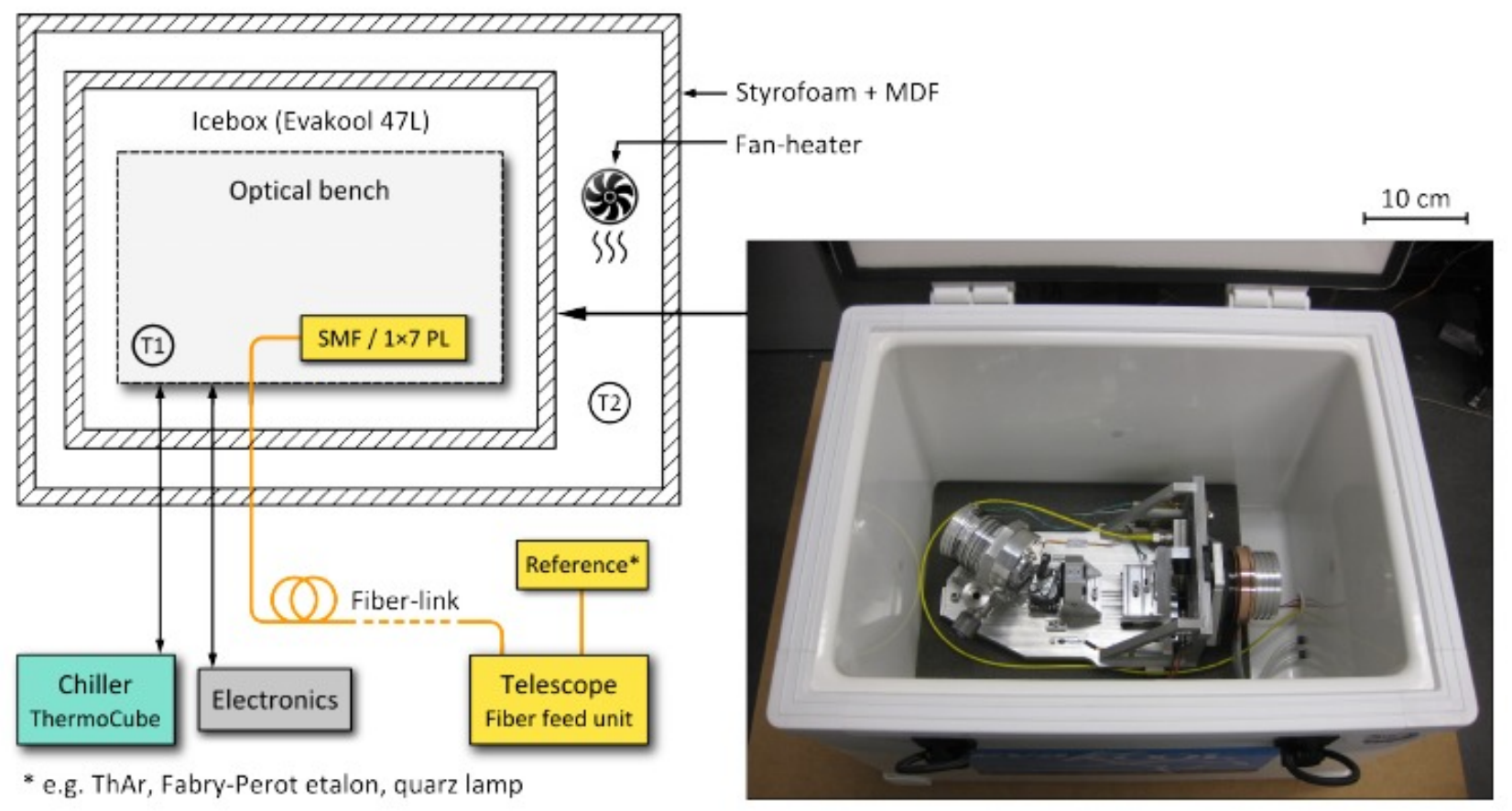

Figure 2. Left: Schematic view of the full instrument enclosures. Right: Photograph of the spectrograph inside the primary enclosure.

order and between successive orders. The test rig consisted of a manual rotation stage, a stabilized laser source (Thorlabs MCLS1-658) which emitted light at $658 \mathrm{~nm}$, a reflective fiber collimator (Thorlabs RC08FC-P01), a SMF (Nufern 460-HP) and a photo-detector (Thorlabs S120C).

We measured the diffracted energy in increments of $0.5^{\circ}$ over a total range of $8^{\circ}$ with respect to the nominal grating orientation $\left(\gamma=0^{\circ}\right)$. The ratio between the power measured before and after diffraction at the échelle is shown in Figure 3 (Left panel). This figure also shows the calculated diffraction angle as a function of the rotation angle (Right panel). At $\beta=64.45^{\circ}$ and rotation angle of $\gamma=5.43^{\circ}$ the laser line approached the blaze maximum of échelle order 86. Considering the reflectivity of bare aluminium we can simply approximate the efficiency for all other wavelengths in the RHEA spectral range.

The measured performance was lower than anticipated with only $27.5 \%$ efficiency at the blaze peak of échelle order 86. Introducing a linear polarizer into the optical beam emerging from the laser did not change the efficiency of the grating. The reason why mass-replicated gratings have such a poor performance can be simply explained by observing the groove profile at high magnification, using a 3D optical surface profiler (Bruker-AXS NT9800). Figure 4 shows an optical profile image of a replicated R2 Thorlabs échelle gratings with $31.6 \mathrm{~g} \mathrm{~mm}^{-1}$. The image indicates irregularities in the stair-like groove profiles which introduce considerable losses.

The optical profile together with the measured efficiency confirm the low performance of mass-replicated échelle gratings. Beveled stair-case profiles scatter the incident light at random angles which increases the background signal in the spectral image and consequently decreases the throughput of the instrument. The optical surface profile indicates that only about $50-60 \%$ of the facet height contributes to light diffraction. Commercially available gratings with higher efficiencies can be purchased e.g. from Newport (Richardson Gratings). échelle gratings of Newport master MR152 (R2, $31.6 \mathrm{~g} \mathrm{~mm}^{-1}$ ), typically have efficiencies on the order of $70-80 \%$ and hence provide a noticeable improvement over inexpensive mass-replicated gratings. We aim to improve the efficiency of RHEA in the future by using such gratings which, once installed in the instrument, can deliver $45 \%$ total efficiency at the blaze maximum (550nm). 

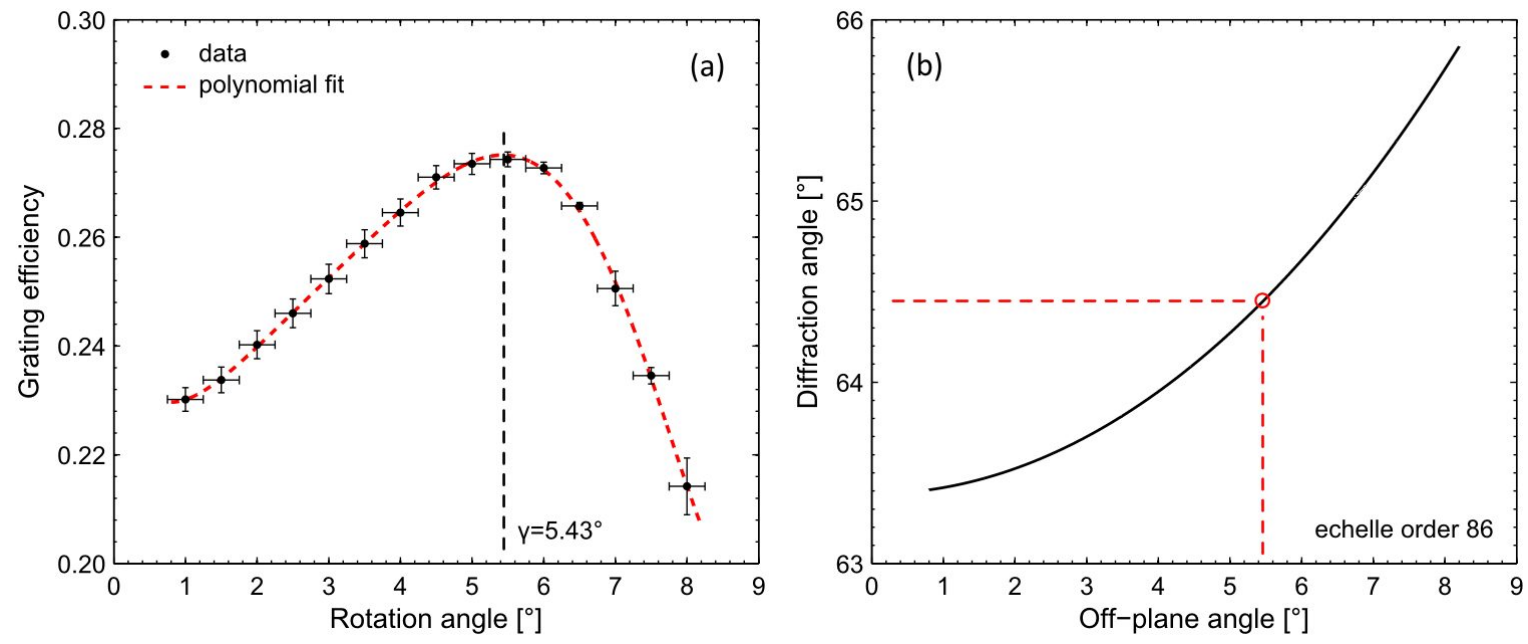

Figure 3. Left panel: Absolute efficiency of a Thorlabs R2 échelle grating (GE2550-0363) as a function of rotation angle $\gamma$, measured at $658 \mathrm{~nm}$. Right panel: Diffraction angle $\beta$ as a function of rotation angle, respectively off-plane angle.

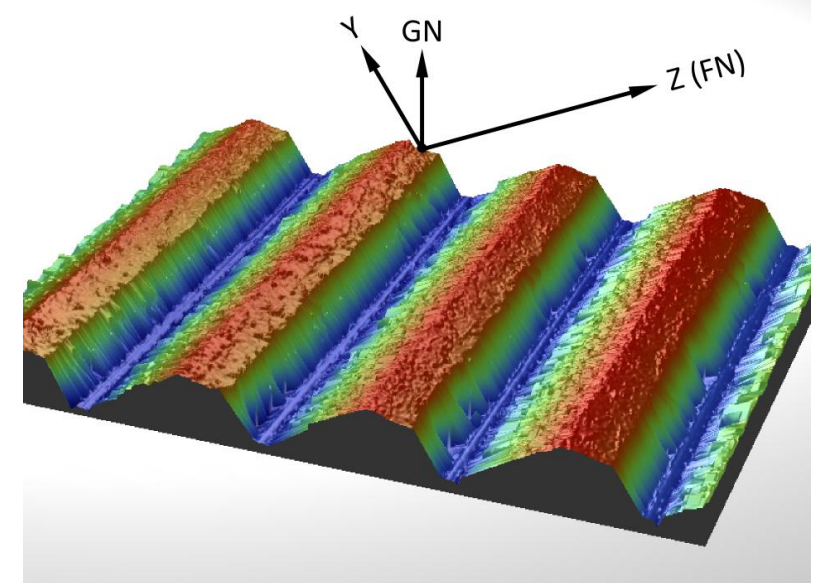

Figure 4. Optical surface profile of a mass-replicated Thorlabs R2 échelle gratings with $31.6 \mathrm{~g} \mathrm{~mm}^{-1}$. The image indicates imperfections in the groove profile. The facet profile along the $\mathrm{Y}$ axis was interpolated and does not reproduce the true roughness of the surface.

\section{ON-SKY PERFORMANCE}

Initial on-sky deployment for this instrument was done at the Macquarie University (MQ) Observatory, situated on the main university campus. Despite being located only $15 \mathrm{~km}$ from the Sydney central area, the proximity to the photonics laboratory where RHEA was developed and the high brightness of test targets makes it the ideal location for rapid prototyping of novel astrophotonics devices.

In this section we describe the first testing phase of this instrument mounted on a 16" Schmidt Cassegrain telescope at the MQ observatory. This telescope is permanently installed on an equatorial SoftwareBisque Paramount ME II mount, which offers precise positioning (periodic peak-to-peak error of 1") and tracking of just over 100kg of instrument payload, making it suitable for single-mode fiber spectroscopy.

We performed observations of various targets in the period of March to December 2015, including RV stable and variable stars as well as the Sun.

All data presented in Section 5.1 were reduced using a custom software pipeline developed by our team aimed at reduction of precision RV measurements from high-resolution multi-fiber input échelle spectrographs. 
This code $^{\dagger}$ is being developed not only for the reduction of RV data from RHEA, but also for the upcoming Gemini High-Resolution Optical SpecTrograph (GHOST), ${ }^{8,9}$ soon to be added to the Gemini South instrument suite and for the upcoming recently funded planet finding spectrograph for the Anglo-Australian telescope at Siding Spring Observatory, Veloce ${ }^{\ddagger}$. Common modules for various tasks are shared between the spectrographs in question, with individual forward optical models for each case.

\subsection{Observations}

In order to test the performance of the RHEA spectrograph in all its science goals, we have selected two test targets aimed at determining the capabilities and limitations of this instrument. We have selected $\tau$ Ceti (HD10700) as an RV stable star where no RV variation should be detected and the Sun as a test bed for nonphoton limited very high S/N observations, which should produce the ultimate test for RV precision of RHEA.

As a test of the overall stability of the spectrograph over a $\sim 10$ day period, we obtained 16 spectra of $\tau$ Ceti, a $\mathrm{V}=3.5$ magnitude main-sequence G-type star, in five separate nights in November 2015. This is a bright RV quiet solar analog in the constellation of Cetus and, as such, has been a target for RV monitoring by several groups. A recent announcement of the suggested presence of a planetary system around this target ${ }^{10}$ from long term RV monitoring data has brought this particular system into the interest of the scientific community, but the low mass of the planets announced would not be detectable by RHEA. Therefore, this is an excellent candidate for on-sky testing.

Figure 5 shows the resulting RV curve for this target as a function of MJD. A downward trend can be seen, indicating that either a systematic error, from an as of yet undetermined nature, is present in the data set or a software reduction artifact may be responsible. We note that nightly data are broadly consistent and that variations happen on a night-to-night basis. This trend is also potentially consistent with errors in the barycentric correction due to inaccurate time stamping of the files. Future observations will likely be undertaken using simultaneous calibration on each individual image in an effort to compensate for such trends (see Section 6)

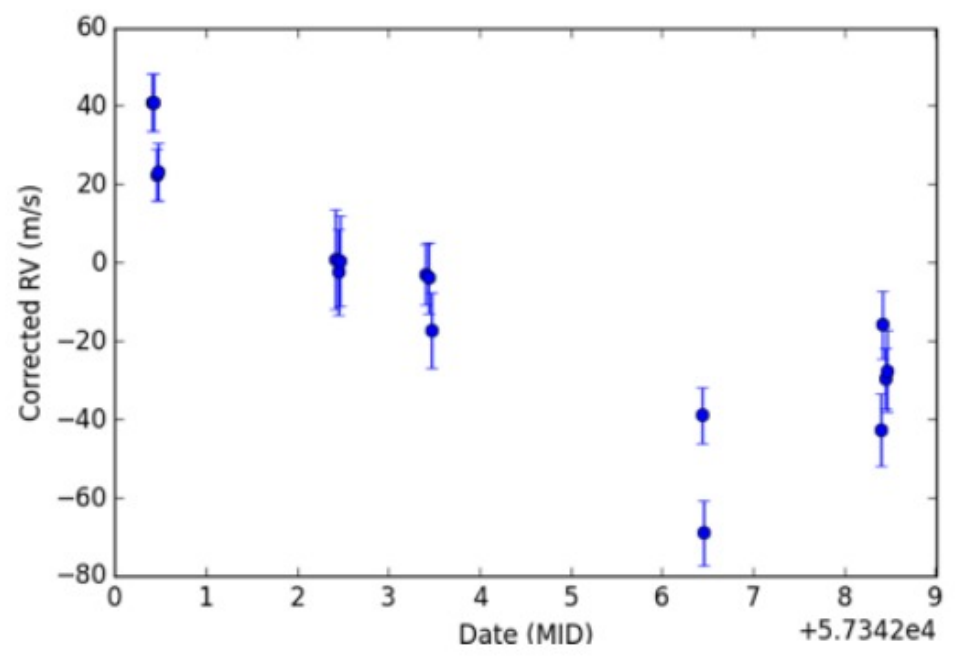

Figure 5. The Radial Velocity signal from $\tau$ Ceti as a function of time for our entire data set. We find a downward trend that requires further investigation.

Solar observations were taken as a test of a key science goal for the RHEA spectrograph. Short term variability in $\mathrm{K}$ giants is the current limitation for finding planets in the later stages of the host star's life. As such, these signals must be detected and eliminated from the data set before signals from planetary mass companions can be searched for. The known acoustic p-mode oscillations of the Sun occur on timescales of $2-4 \mathrm{mHz}$ with the

\footnotetext{
${ }^{\dagger}$ Available at https://github.com/mikeireland/pymfe

${ }^{\ddagger}$ More information on this instrument at http://newt.phys.unsw.edu.au/ cgt/Veloce/Veloce.html
} 
most prominent features at $3.3 \mathrm{mHz}(\sim 5$ minutes). Therefore, RV sampling at much faster rate is required for a conclusive analysis.

Several observing runs were performed over 2015 and we selected the longest for the basis of this analysis. 1075 spectra were obtained over a four hour period with 1.1 second exposures leading to a sampling rate of 13 seconds due to CCD readout time. Observations were interrupted once per hour for a series of ThAr calibration frames to be acquired. These data were acquired using a modified method from that for point source targets, since using the full telescope aperture would have resulted in damage to the fibers. A $3.5 \mu \mathrm{m}$ core fiber was pointed directly at the solar disk, mounted on the side of the telescope OTA, with an acceptance angle that easily integrates the full solar disk and surrounding halo. This fiber was then fed into a $2 \times 2$ single-mode fiber coupler with a 50/50 split ratio, the output of which was then fed into the RHEA spectrograph. The ThAr source was then injected into the second input of the coupler to ensure that there was no need to detach any fibers during calibration frames.

A sub-set of the data taken is shown in Figure 6 (Top panel), where a two hour long run is displayed. In order to reduce individual data uncertainties we have chosen to bin our data points as well as smooth the data set using a Savitzky-Golay filter ${ }^{11}$ to enhance the oscillation signal and remove the high frequency noise.
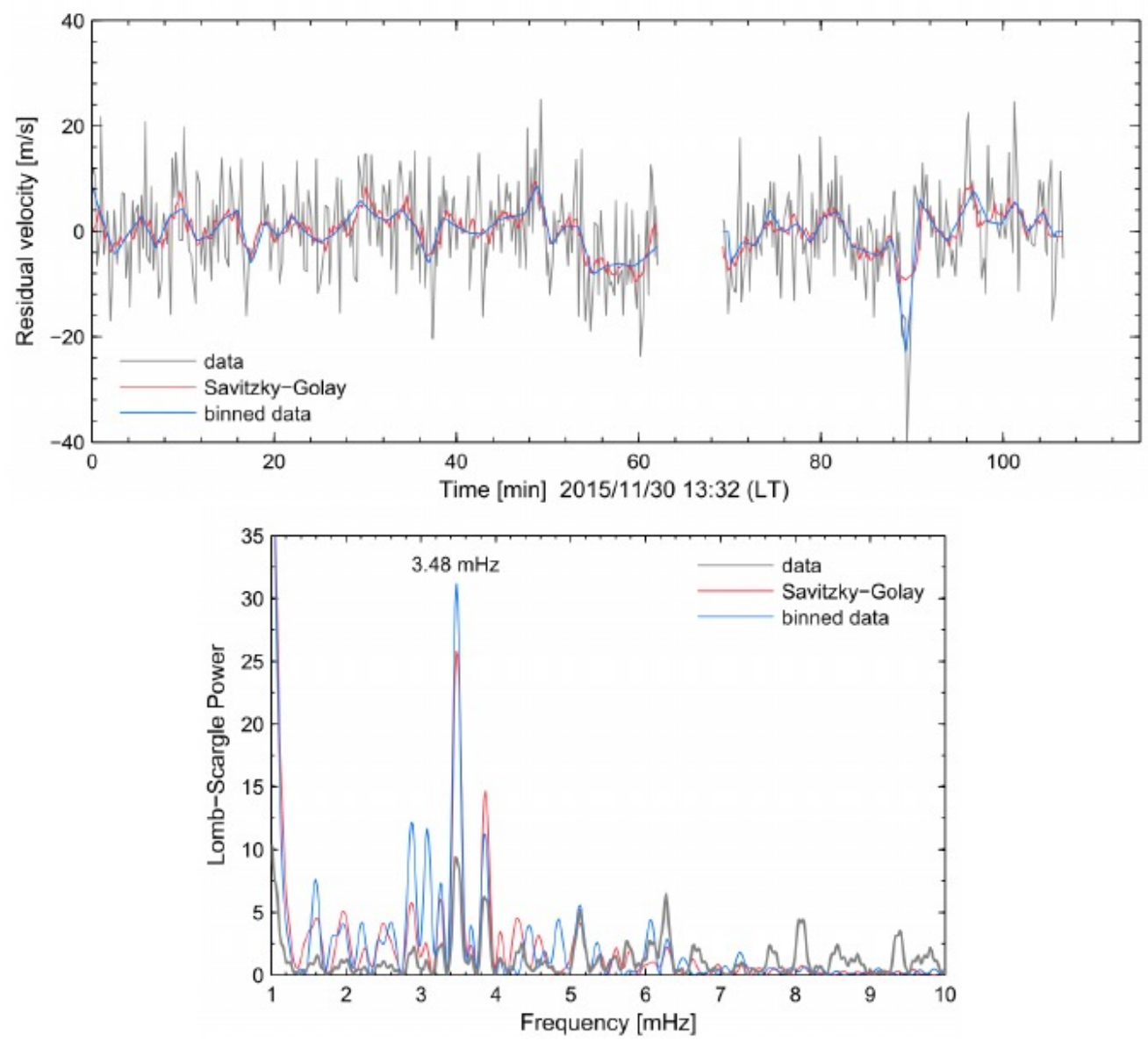

Figure 6. Top: A 2 hour subset of the Radial Velocity signal from Solar observations as a function of time. Bottom: Lomb-scargle periodogram from our data set (blue) as a comparison with the previous known result (black). We tentatively recover the two lowest period signals.

Despite a significant presence of high frequency noise still in the Lomb-Scargle periodogram (Figure 6 Bottom panel), we do clearly recover a significant peak at 288 seconds (4.8 minutes) corresponding to the predominant solar oscillation period. This variation is seen on a much faster timescale than any instrumental drift thereby 
confirming the origin of such RV variability, thereby also confirming that RHEA is capable of high-precision RV monitoring at the few $\mathrm{m} / \mathrm{s}$ level.

\section{IMPROVEMENTS UNDER DEVELOPMENT AND FUTURE WORK}

At this point in time the next major steps in the RHEA project naturally lead to two objectives: Longer baseline on-sky observations and optical throughput improvements. In this section we will address the planned and ongoing efforts in this context.

A first effort at very long baseline observations with RHEA will be addressed with the upcoming commissioning of the spectrograph at a dedicated already existing dome located at ANU's Mount Stromlo Observatory on the edge of Canberra, Australia. This site has better weather statistics and lower light pollution levels than the previous on-sky testing site at the Macquarie University campus. Moreover, the facility will be dedicated uniquely to this purpose and will be fully automated, allowing for maximum efficiency observations. The spectrograph will be fed by a Bintel Ritchey-Chrétien design GSO RC10-A 10" aperture Astrograph mounted on a SkyWatcher NEQ6 pro equatorial mount. This setup was chosen in the same mindset of inexpensive replicable units, allowing for a full cost of under AUD $\$ 6,000$ for telescope and mount. We aim to have the first on-sky observations from Mount Stromlo in September 2016.

Much like the system deployed at the MQ observatory we will use a fiber feed optical system to inject starlight onto the single mode fiber. However, despite the fact that this will be a similar optical design to that used previously, we plan to implement some fundamental changes that will improve coupling and throughput.

The fundamental issue of using single-mode fibers vs multi-mode equivalents is the reduced core diameter that then leads naturally to significantly reduced fraction of light coupled into the fiber. In order to address this issue, we will be injecting the stellar light onto a square format array of single-mode fibers that will then be reformatted onto a linear structure for input on the spectrograph. This method is inspired by the photonic lantern concept, ${ }^{12,13}$ which has been used for multiple applications, including on-chip multi-mode to single-mode coupling with in-fiber $\mathrm{OH}$ suppression (e.g. ${ }^{14}$ ).

In order to achieve this purpose, we have fabricated a custom fiber cable where we have a $3 \times 3$ arrangement of single-mode fibers separated by $1 \mathrm{~mm}$ being fed simultaneously through a micro-lens array with $4.64 \mathrm{~mm}$ focal length lenses that focuses the input starlight onto each fiber individually. With this system we have essentially developed an integral field unit (IFU) for our spectrograph.

Figure 7 shows photos of this component. We have used two glass prisms to connect the microlens array and optical fibers, ensuring that these are glued to the back surface of the prisms at the correct focal length. The use of these two prisms is an elegant way to allow for optical alignment and correct spacing between the lens array and the fibers. Each fiber was stripped and glued to the inside of a metal ferrule which was then polished as a preparation for the gluing to the prism. We then used a 6 axis stage to move each fiber into the correct position. This was ensured by back illuminating all the fibers and imaging both the near and far fields of the microlens array such that they overlap. As such, small angle tilts with respect to the perpendicular direction on the prism surface are required for all fibers other than the central fiber. UV curing glue was then used to bond the fibers to the surface individually and more was used on the full bundle for added strength. Finally, a metal piece was inserted that surrounds all ferrules and provides strain relief for the full cable. The number of single-mode fibers that are glued depends on the design for each individual copy of the RHEA spectrograph, since the spacing between orders on the chip is wavelength dependent. We note that 9 fibers were used for the version deployed at the Subaru Telescope as the cross dispersion adopted for that particular version was higher but only 7 fibers will be available for the version we describe in this paper.

As mentioned earlier, this component is responsible for re-formatting the $3 \times 3$ arrangement onto a linear format that is then injected onto the spectrograph. As such, the end of the bundle of fibers is then placed side by side on a V-groove mount and glued in place. At this stage we have elected to place an additional fiber which will be used for calibration purposes, either wavelength reference source or flat-field lamp. Critically, the wavelength reference source will be used simultaneously with every exposure thereby providing a direct measurement of the wavelength scale for each spectra without the need for temporal interpolation and eliminating the need for 

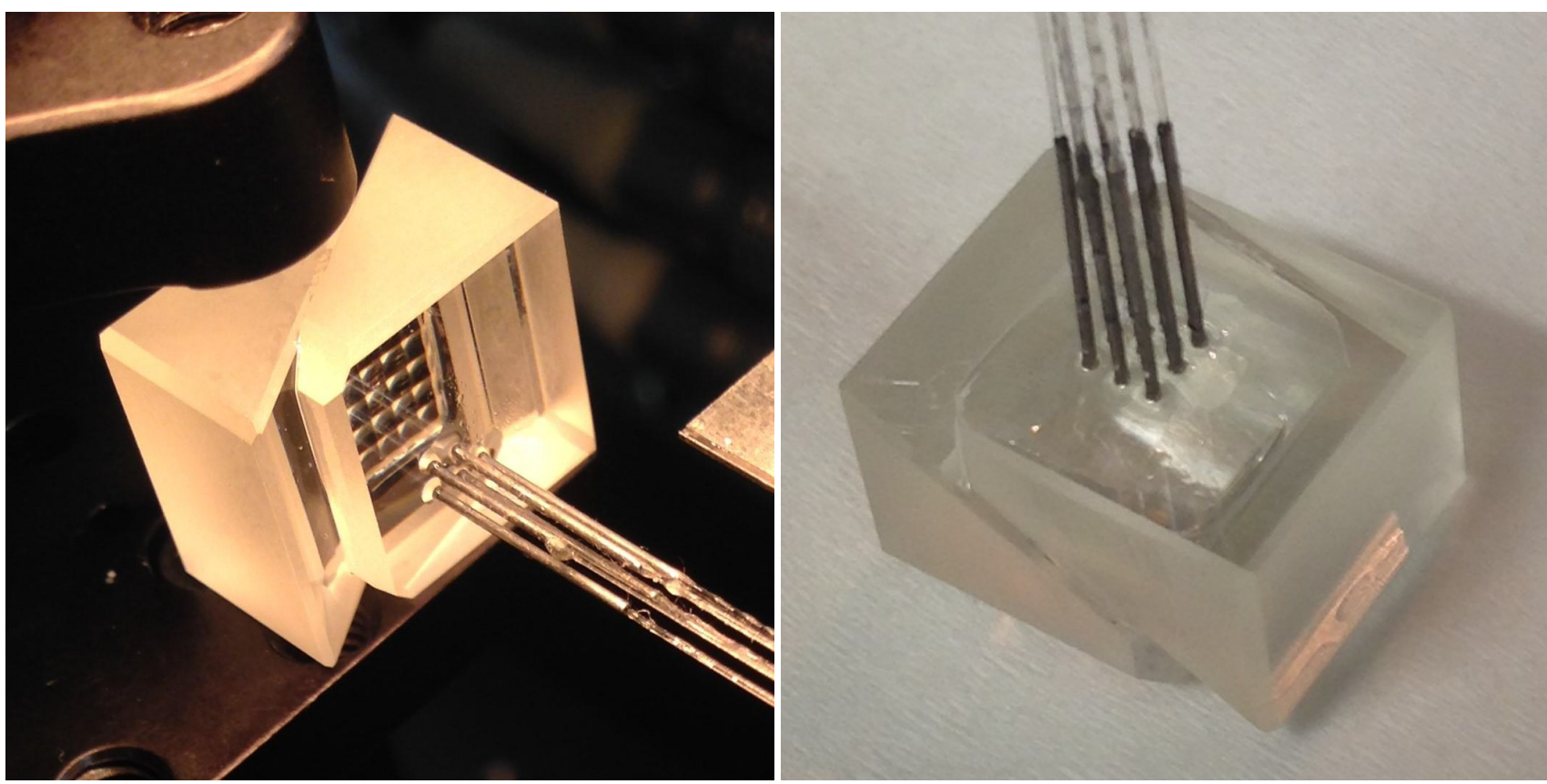

Figure 7. Left: RHEA IFU under fabrication. The microlens array can be seen through the glass prisms. Right: Back view of the RHEA IFU with all fiber ferrules glued to the glass prisms.

intermittent calibrations. This should maximize the RV precision achievable for this instrument as we do not expect significant variations in spectrograph stability during single exposures (typically 30-60 minutes).

The linear format output of the fiber bundle is then bonded to a linear microlens array as part of the slit optics of the spectrograph. Light has been successfully simultaneously injected into all fibers and a full characterization of the throughput and efficiency of this device will be done soon.

In addition to this fiber IFU we aim to increase the light coupling efficiency by addressing the typical seeing conditions at the typical sites that copies of RHEA are likely to be commissioned in. We are therefore in the process of developing and building a custom made inexpensive and small tip/tilt system comprised of 2 voice coil actuators pushing on a lens mount to move the lens in translation. This will be used in a closed loop in which the acquisition camera will serve as the feedback source. Additionally, in order to minimize the effect of the telescope's central obstruction we aim to install a set of optics designed to produce Phase Induced Aperture Apodization (PIAA) ${ }^{15}$ and therefore reshape the incoming PSF. This is a beam shaping technique that also enhances the coupling into single-mode fibers.

We have modeled the predicted effects of these two components by producing a realistic model of the atmospheric turbulence with varying seeing conditions with the introduction of both a tip/tilt system and PIAA optics. Displayed in Figure 8 is a result from our simulations where we show the gain factor in terms of the amount of light that can be successfully coupled into the fibers for three cases: a single fiber, 5 fibers (in a cross arrangement) and all 9 fibers. We find that, for a typical seeing of 2" we should achieve a gain of $\sim 1.7$ for the full IFU implementation. Naturally, the use of an IFU alone already compensates partially for the effects of seeing but we also show that we will be improve our system through the use of both a tip/tilt and PIAA optics system significantly. This will allow us to observe fainter targets and increase the numbers of possible candidates for long baseline observations with RHEA since we will be able to collect more light than a single fiber case.

\section{SUMMARY}

In this paper we present the current status of the RHEA spectrograph as well as results from additional lab tests and preliminary on-sky performance. We show that this inexpensive instrument is capable of achieving few $\mathrm{m} / \mathrm{s}$ RV stability in a laboratory environment and that the throughput performance of our current prototype 


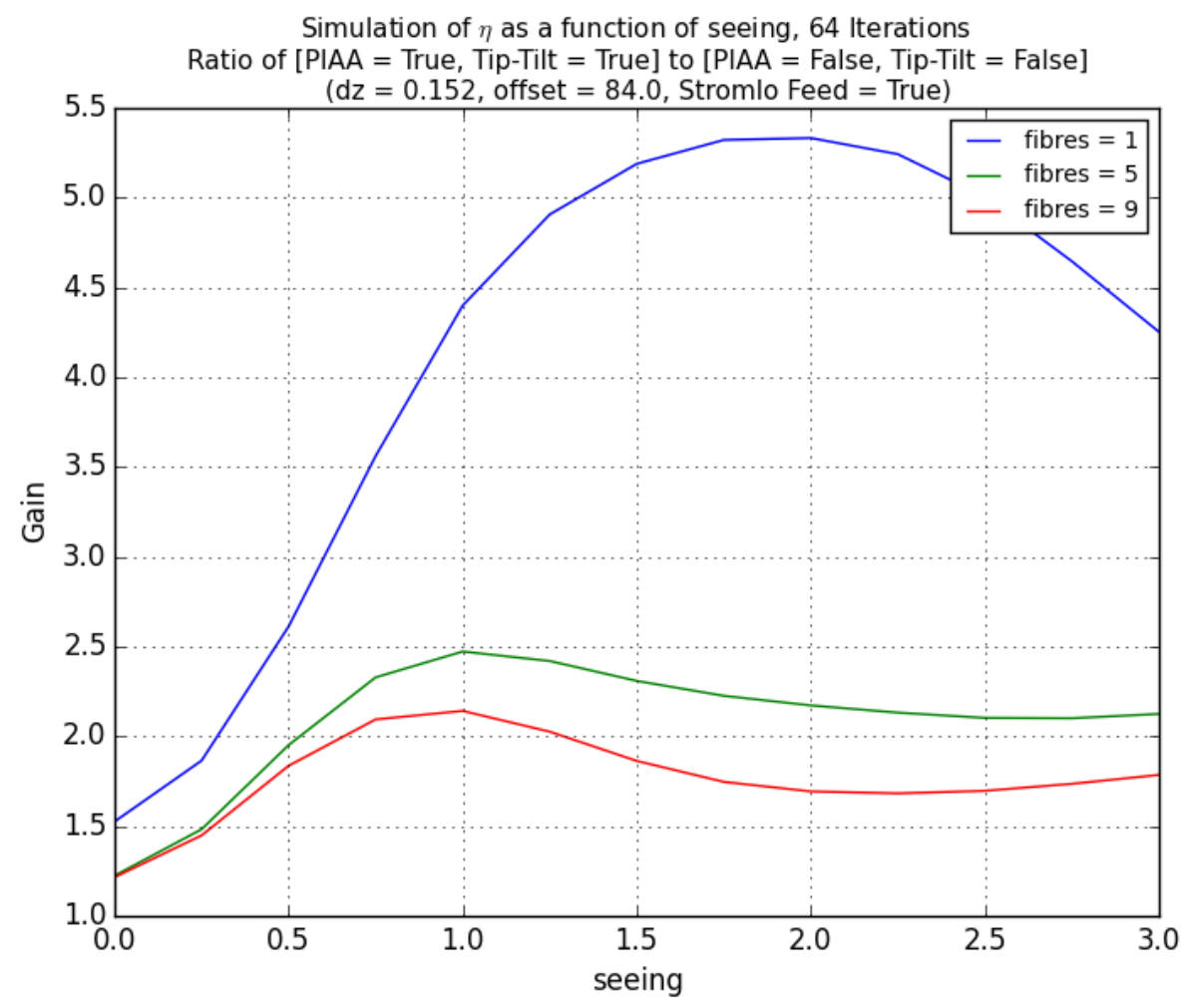

Figure 8. Light coupling factor as a function of seeing. This gain represents the gain in terms of light successfully coupled into the optical fibers between using both PIAA optics and tip/tilt system and neither. We find an increase of at least $60 \%$ across the full range of expected seeing conditions ( $>1$ ") for all 9 fibers used. This setup is particularly impressive when a single fiber case is considered.

is limited by the low efficiency of the échelle grating currently commissioned. Future improvements include the installation and testing of higher quality options for this component.

We describe the method and results from the first set of on-sky observations with this spectrograph, commissioned on the Macquarie University Observatory 16" telescope. A series of observations of a nearby RV quiet solar analog target $(\tau$ Ceti) revealed the presence of a trend thought to be likely related to an artifact in the processing pipeline or an unknown systematic factor. We hope to address this issue with simultaneous calibration in future observations which will enable us to better handle software pipeline issues.

We also present observations of the solar spectrum with 13 second sampling over a four hour run, in which we clearly recover the predominant p-mode solar 5-minute oscillation. This result confirms that RHEA can indeed perform under the stringent conditions of typical small robotic telescopes at the level required for Jupiter sized exoplanet detection via the RV method.

We also discuss the future steps to improve and indeed shape this instrument for a full deployment on multiple sites. We discuss the implementation of a modified fiber feed with a custom tip/tilt and Phase Induced Amplitude Apodisation (PIAA) optics to dramatically improve throughput and thus widen the range of possible targets to higher magnitudes. We plan to also use an IFU injection system, akin to a photonic lantern, for the same purpose. We find that we can obtain over $60 \%$ gain in light coupling efficiency by introducing such improvements. Future on-sky tests with simultaneous wavelength calibration will reveal the true improvement in both efficiency and RV precision of this instrument.

\section{ACKNOWLEDGMENTS}

This work was partially funded by the Australian Research Council Discovery Project DP120103751. We thank the Macquarie University Engineering and Technical Services and ANU's Research School of Astronomy and 
Astrophysics mechanical workshop for the work in manufacture of critical parts for RHEA. We thank Martin Ams and Benjamin Johnston for the use of the OptoFab photonics laboratory and useful input in the construction of the fiber cable bundle.

\section{REFERENCES}

[1] McCoy, K. S., Ramsey, L., Mahadevan, S., Halverson, S., and Redman, S. L., "Optical fiber modal noise in the 0.8 to 1.5 micron region and implications for near infrared precision radial velocity measurements," in [Ground-based and Airborne Instrumentation for Astronomy IV], Proc. SPIE 8446, 84468J (Sept. 2012).

[2] Mahadevan, S., Halverson, S., Ramsey, L., and Venditti, N., "Suppression of Fiber Modal Noise Induced Radial Velocity Errors for Bright Emission-line Calibration Sources," ApJ 786, 18 (May 2014).

[3] Halverson, S., Roy, A., Mahadevan, S., and Schwab, C., "'Modal Noise" in Single-mode Fibers: A Cautionary Note for High Precision Radial Velocity Instruments," ApJ 814, L22 (Dec. 2015).

[4] Rains, A., Ireland, M. J., Jovanovic, N., Bento, J., Feger, T., Schwab, C., Arriola, A., Coutts, D., and Guyon, O., "Precision single mode fibre integral field spectroscopy with the RHEA spectrograph," in [Ground-based and Airborne Instrumentation for Astronomy IV], Proc. SPIE 9908 (Sept. 2016).

[5] Bernstein, R., Shectman, S. A., Gunnels, S. M., Mochnacki, S., and Athey, A. E., "MIKE: A Double Echelle Spectrograph for the Magellan Telescopes at Las Campanas Observatory," in [Instrument Design and Performance for Optical/Infrared Ground-based Telescopes], Iye, M. and Moorwood, A. F. M., eds., Proc. SPIE 4841, 1694-1704 (Mar. 2003).

[6] Crane, J. D., Shectman, S. A., and Butler, R. P., "The Carnegie Planet Finder Spectrograph," in [Society of Photo-Optical Instrumentation Engineers (SPIE) Conference Series], Proc. SPIE 6269, 626931 (June 2006).

[7] Feger, T., Bacigalupo, C., Bedding, T. R., Bento, J., Coutts, D. W., Ireland, M. J., Parker, Q. A., Rizzuto, A., and Spaleniak, I., "RHEA: the ultra-compact replicable high-resolution exoplanet and Asteroseismology spectrograph," in [Ground-based and Airborne Instrumentation for Astronomy V], Proc. SPIE 9147, 91477I (Aug. 2014).

[8] Ireland, M. J., Barnes, S., Cochrane, D., Colless, M., Connor, P., Horton, A., Gibson, S., Lawrence, J., Martell, S., McGregor, P., Nicolle, T., Nield, K., Orr, D., Robertson, J. G., Ryder, S., Sheinis, A., Smith, G., Staszak, N., Tims, J., Xavier, P., Young, P., and Zheng, J., "The AAO's Gemini High-Resolution Optical SpecTrograph (GHOST) concept," in [Ground-based and Airborne Instrumentation for Astronomy IV], Proc. SPIE 8446, 844629 (Sept. 2012).

[9] Ireland, M., Anthony, A., Burley, G., Chisholm, E., Churilov, V., Dunn, J., Frost, G., Lawrence, J., Loop, D., McGregor, P., Martell, S., McConnachie, A., McDermid, R. M., Pazder, J., Reshetov, V., Robertson, J. G., Sheinis, A., Tims, J., Young, P., and Zhelem, R., "Progress on the Gemini High-Resolution Optical SpecTrograph (GHOST) design," in [Ground-based and Airborne Instrumentation for Astronomy V], Proc. SPIE 9147, 91471J (July 2014).

[10] Tuomi, M., Jones, H. R. A., Jenkins, J. S., Tinney, C. G., Butler, R. P., Vogt, S. S., Barnes, J. R., Wittenmyer, R. A., O'Toole, S., Horner, J., Bailey, J., Carter, B. D., Wright, D. J., Salter, G. S., and Pinfield, D., "Signals embedded in the radial velocity noise. Periodic variations in the $\tau$ Ceti velocities," $A \mathscr{E} A$ 551, A79 (Mar. 2013).

[11] Savitzky, A. and Golay, M. J. E., "Smoothing and differentiation of data by simplified least squares procedures.," Analytical Chemistry 36(8), 1627-1639 (1964).

[12] Leon-Saval, S. G., Birks, T. A., Bland-Hawthorn, J., and Englund, M., "Multimode fiber devices with single-mode performance," Opt. Lett. 30, 2545-2547 (Oct 2005).

[13] Noordegraaf, D., Skovgaard, P. M. W., Nielsen, M. D., and Bland-Hawthorn, J., "Efficient multi-mode to single-mode coupling in a photonic lantern," Opt. Express 17, 1988-1994 (Feb 2009).

[14] Spaleniak, I., Gross, S., Jovanovic, N., Williams, R. J., Lawrence, J. S., Ireland, M. J., and Withford, M. J., "Multiband processing of multimode light: combining 3D photonic lanterns with waveguide Bragg gratings," ArXiv e-prints (Nov. 2013).

[15] Guyon, O., "Phase-induced amplitude apodization of telescope pupils for extrasolar terrestrial planet imaging," $A \mathscr{G} A$ 404, 379-387 (June 2003). 